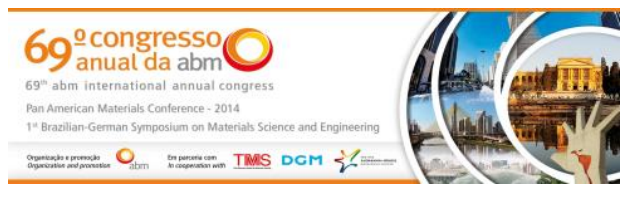

Tema: Iniciação científico-tecnológica

\title{
ESTUDO DA UTILIZAÇÃO DE ÓLEOS VEGETAIS E INTEGRAL COMO FLUIDO DE CORTE NO PROCESSO DE RETIFICAÇÃO*
}

\author{
Leonardo Roberto da Silva \\ Felipe Amaral Oliveira ${ }^{2}$ \\ Evanilton José Alves Barbosa ${ }^{3}$ \\ Helane Lúcia Oliveira de Morais ${ }^{3}$ \\ Claudinei Rezende Calado 4 \\ Cintia Braga ${ }^{5}$ \\ Izabela Silva Bicalho
}

\section{Resumo}

Os fluidos de corte são empregados no processo de retificação com o intuito de lubrificar e refrigerar a peça/rebolo, além de amenizar problemas que afetam a integridade superficial da peça e também melhoram a eficiência do processo. Os fluidos de corte são muito utilizados na retificação, porém são tóxicos, dificilmente degradáveis e nocivos ao meio ambiente. Dessa maneira, é pertinente a formulação de fluidos menos agressivos. Neste trabalho, avaliou-se o desempenho de óleos vegetais como fluidos de corte em comparação com o fluido de corte integral no processo de retificação do aço ABNT 4340 temperado e revenido com dureza média de 52 HRc. O desempenho dos fluidos consistiu na análise de rugosidade, formação de espuma e odor. A caracterização dos óleos foi realizada pela viscosidade, densidade, ponto de fulgor, corrosão, índice de acidez total, índice de iodo e de refração. Os resultados mostraram que as peças retificadas com óleos vegetais apresentaram rugosidades próximas às peças usinadas com fluido integral. $\mathrm{Na}$ caracterização, os óleos vegetais apresentaram maior viscosidade que o integral e suas densidades foram próximas. No resfriamento, os óleos vegetais foram capazes de resfriar mais as peças que o integral, além de corroer menos. Nos outros aspectos analisados, o óleo de soja foi o que apresentou melhor desempenho. Esses resultados comprovam a eficiência dos óleos vegetais e viabilidade do uso no processo.

Palavras-chave: Óleos vegetais; Fluidos de corte; Processo de retificação; Manufatura ecologicamente correta.

\section{STUDY ON THE UTILIZATION OF VEGETAL AND INTEGRAL OILS USED AS CUTTING FLUID IN GRINDING PROCESS}

\section{Abstract}

Cutting fluids are used in the grinding process in order to lubricate and cool the piece/grinding wheel, besides all aviate problems that affect the surface integrity of the piece and also improve the efficiency of the process. Cutting fluids are widely used in the grinding process, but are toxic, hardly degradable and environmental hazard. Thus, it is pertinent the formulation of less aggressive fluids. This study evaluated the performance of vegetable oils as cutting fluids compared with the integral fluid cutting in the grinding process of the hardened AISI 4340 steel with average hardness of 52 HRc. The performance of the fluids was based in the analysis of the surface roughness, temperature, foaming and odor. The characterization of the oils was performed by viscosity, density, flash point, corrosion, index total acidity, iodine value and refractive. The results showed that the pieces rectified with vegetable oils presented close values of surface rougness the machined pieces with the integral fluid. In characterization, vegetable oils showed higher viscosity than the integral and their densities were close. On cooling process, the vegetable oils were able to cool down more than the integral, in addition to corrode less. In other analyzed aspects, soybean oils howed the best performance. These results prove the efficiency of vegetable oils and the feasibility of using in the process.

Keywords: Vegetable oils; Cutting fluids; Grinding process; Eco-Friendly Manufacturing.

1 Engenharia Mecânica, Doutor, Coordenador do Mestrado em Engenharia de Materiais, DEMAT, CEFET-MG, Belo Horizonte, Minas Gerais, Brasil.

2 Engenharia de Materiais, Graduando, Departamento de Engenharia de Materiais, CEFET-MG, Belo Horizonte, Minas Gerais, Brasil.

${ }^{3}$ Engenharia de Materiais, Mestrando, Departamento de Engenharia de Materiais, CEFET-MG, Belo Horizonte, Minas Gerais, Brasil.

${ }^{4}$ Química, Doutor, Professor, Departamento de Química, CEFET-MG, Belo Horizonte, Minas Gerais, Brasil.

${ }^{5}$ Engenharia de Materiais, Graduando, Departamento de Engenharia de Materiais, CEFET-MG, Belo Horizonte, Minas Gerais, Brasil.

\footnotetext{
* Contribuição técnica ao 69 Congresso Anual da ABM - Internacional e ao $14^{\circ}$ ENEMET - Encontro Nacional de Estudantes de Engenharia Metalúrgica, de Materiais e de Minas,21 a 25 de julho de 2014, São Paulo, SP, Brasil.
} 


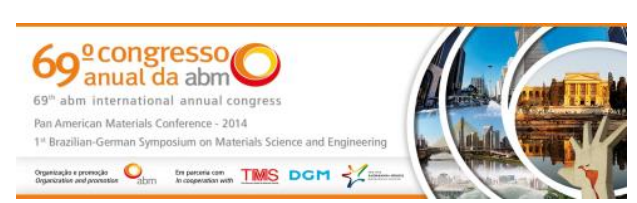

\section{INTRODUÇÃO}

Os efeitos positivos da utilização de fluidos de corte foram verificados por Taylor em 1894, onde ao aplicar uma determinada quantidade de água na área de corte percebeu que a velocidade de corte poderia ser aumentada em $33 \%$ sem a redução da vida útil da ferramenta [1]. Desde então as operações de usinagem passaram por constantes evoluções. Além do aumento da velocidade de corte e da redução do desgaste da ferramenta outras características são apresentadas por Maia et al. [2], as quais são:

- Refrigeração e lubrificação;

- Melhora no acabamento da superfície;

- Proteção contra corrosão;

- Remoção das aparas da peça por ação de lavagem;

- Redução do consumo de potência (energia).

Não existe hoje no mercado um único tipo de fluido que atenda todas as necessidades operacionais. $O$ que existe são fluidos mais versáteis que podem ser aplicados em diferentes tipos de operações [3]. Historicamente, observa-se que os fluidos de corte são, na maioria das vezes, à base de óleo mineral, devido principalmente à relação entre preço e características técnicas solicitadas. No entanto os efeitos negativos gerados quanto à contaminação dos recursos hídricos, solo e o ambiente em geral explicam a necessidade de estudos sobre o seu uso em demasia [4].

Uma alternativa seria a aplicação de óleos vegetais na forma de fluidos de corte, uma vez que além de serem de fonte renovável, apresentam alto poder lubrificante e é uma matéria prima abundante no Brasil na produção de soja, milho, amendoim, algodão, babaçu e palma [5-6]. Segundo Gonçalves [7], quanto maior a necessidade de lubrificação, maior a vantagem de utilização de fluidos de corte de base vegetal em relação às demais bases, independente da condição de corte empregada.

Estudos sobre a viabilidade e vulnerabilidade de óleos vegetais para utilização e substituição como fluido de corte devem estar direcionados de forma a abranger as seguintes áreas: biológicas (esclarecer relações benéficas da biodegradação na ação microbiológica do fluido), química (reações tribológicas da usinagem com o fluido) e mecânica (relação fluido/ferramenta). No entanto, até o presente momento, poucos estudos referentes ao comportamento reológico de óleos vegetais têm sido reportados na literatura [8].

Com o intuito de desenvolver um processo mais sustentável o presente trabalho realizou a incorporação de óleos vegetais numa matriz experimental de fluido de corte. Essa incorporação considerou os fatores econômicos, técnicos e ambientais para viabilizar esta matriz. Além disso, essa matriz foi avaliada e caracterizada no processo de retificação visando monitorar seu desempenho como fluido de corte. (Características lubrificantes, refrigeradoras e comportamento oxidativo).

\section{MATERIAIS E MÉTODOS}

A avaliação do desempenho dos óleos vegetais, mineral e suas combinações, foi baseada de acordo com as seguintes propriedades: viscosidade, densidade, ponto de fulgor, corrosão, índice de acidez total, índice de iodo e de refração. Durante a retificação observou-se ainda os parâmetros de temperatura, formação de fumaça,

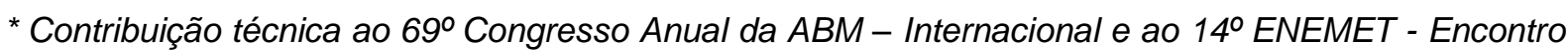
Nacional de Estudantes de Engenharia Metalúrgica, de Materiais e de Minas,21 a 25 de julho de 2014, São Paulo, SP, Brasil.
} 
espuma e odor, visando comparar o comportamento dos óleos vegetais e suas formulações com o fluido de corte comercial (óleo mineral).

O desenvolvimento deste trabalho foi realizado por uma retificadora cilíndrica universal (operação externa de mergulho), da marca TosHostivar, onde foram confeccionados corpos de prova de aço ABNT 4340 endurecido com dureza média de 52 HRc.

Foram utilizados os seguintes equipamentos:

- Reficadora cilíndrica universal TosHostivar (Figura 1);

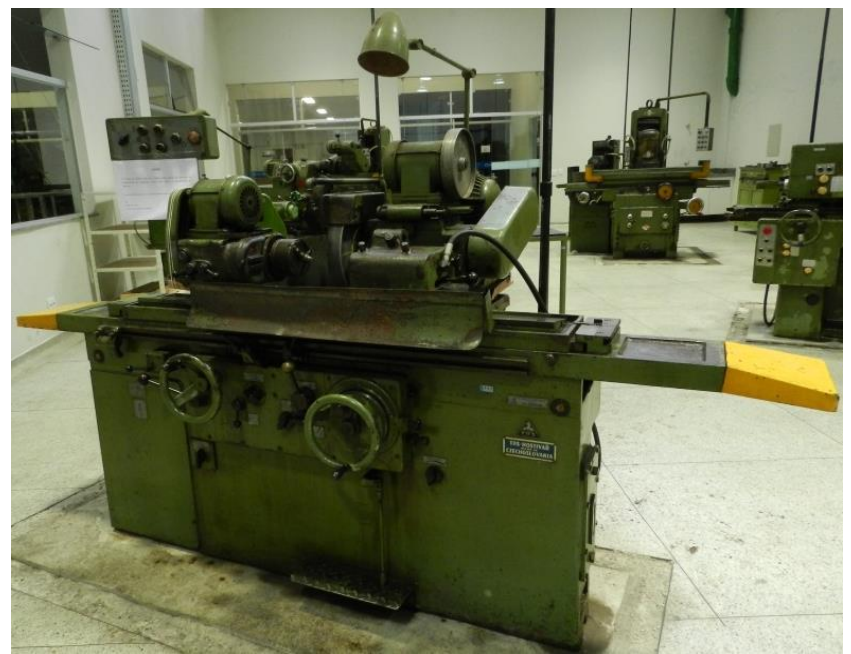

Figura 1. Reficadora cilíndrica universal Tos Hostivar.

- Termômetro infravermelho, fabricante Minipa, modelo MT-390;

- Rugosímetro Mitutoyo, modelo SJ-301;

- Agitador mecânico, modelo TE 139 - Tecnal;

- Refratômetro;

- Aparelho ponto de fulgor Vaso Aberto Cleveland, marca Quimis;

- Viscosímetro Brokfield, modelo DV II+Pró.

Os óleos utilizados foram:

- Óleo mineral de base parafínica Mecafluid 14SC, marca Petronas Lubrificantes do Brasil de base parafínica;

- Óleos vegetais de soja (Glycinemax) e milho (Zeamays), do fabricante Campestre.

A Tabela 1 apresenta as normas segundo as quais foram realizados os ensaios de caracterização físico-química dos óleos e rugosidade da peça.

Tabela 1. Técnicas utilizadas nos ensaios realizados

\begin{tabular}{l|l}
\hline \multicolumn{1}{c}{ Ensaio } & \multicolumn{1}{c}{ Norma } \\
\hline Índice de Acidez & ASTM D974 \\
\hline Índice de lodo & Metodologia do Instituto Adolfo Lutz \\
\hline Índice de Refração & Metodologia do Instituto Adolfo Lutz \\
\hline Ponto de Fulgor & ASTM D92 \\
\hline Corrosão à Lâmina de Cobre & NBR 10505 \\
\hline Densidade & ASTM D1298 \\
\hline Viscosidade & ASTM D2770-4 \\
\hline Rugosidade & DIN 4776 \\
\hline
\end{tabular}

\footnotetext{
* Contribuição técnica ao 69ำ Congresso Anual da ABM - Internacional e ao 14ํㅡㄹ ENEMET - Encontro Nacional de Estudantes de Engenharia Metalúrgica, de Materiais e de Minas,21 a 25 de julho de 2014, São Paulo, SP, Brasil.
} 


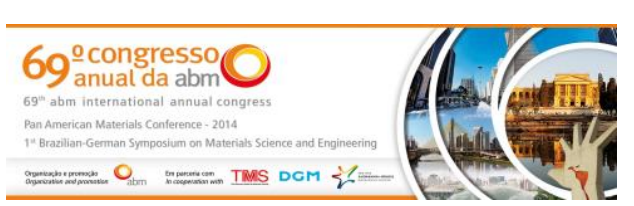

Tabela 2. Resultados da caracterização físico-química dos óleos.

\begin{tabular}{|c|c|c|c|}
\hline Ensaio & Óleo Mineral & Óleo de Soja & Óleo de Milho \\
\hline Índice de Acidez $\left(\mathrm{mgKOH}_{\mathrm{g}} \mathrm{g}^{-1}\right)$ & $2,11 \pm 0,04$ & $0,210 \pm 0,002$ & $0,310 \pm 0,003$ \\
\hline İndice de lodo $\left(\mathrm{gl}_{2 \cdot \mathrm{g}^{-1}}\right)$ & 10,8 & 108,0 & 78,6 \\
\hline Índice de Refração & $1,48 \pm 0,02$ & $1,4740 \pm 0,02$ & $1,48 \pm 0,02$ \\
\hline Ponto de Fulgor $\left({ }^{\circ} \mathrm{C}\right)$ & 174 & 328 & 320 \\
\hline Corrosão à Lâmina de Cobre & $4 \mathrm{~b}$ & $1 \mathrm{a}$ & $1 \mathrm{a}$ \\
\hline Densidade $\left(\mathrm{g} \cdot \mathrm{cm}^{-3}\right)$ & 0,8777 & 0,9181 & 0,9181 \\
\hline Viscosidade $(\mathrm{cSt})$ & 12,65 & 29,70 & 34,73 \\
\hline Rugosidade $-\mathrm{Ra}(\mu \mathrm{m})$ & $0,17 \pm 0,03$ & $0,18 \pm 0,04$ & $0,23 \pm 0,02$ \\
\hline
\end{tabular}

Os valores obtidos no ensaio de índice de acidez apresentaram valores muito próximos para os óleos vegetais. Já o óleo de origem mineral apresentou um índice de acidez muito superior aos óleos vegetais.

A probabilidade de um óleo se comportar como um meio corrosivo é diretamente proporcional ao seu índice de acidez. Assim o ensaio de corrosão à lâmina de cobre comprovou este comportamento uma vez que o óleo mineral apresentou um grau de corrosão muito superior (Figura 4).
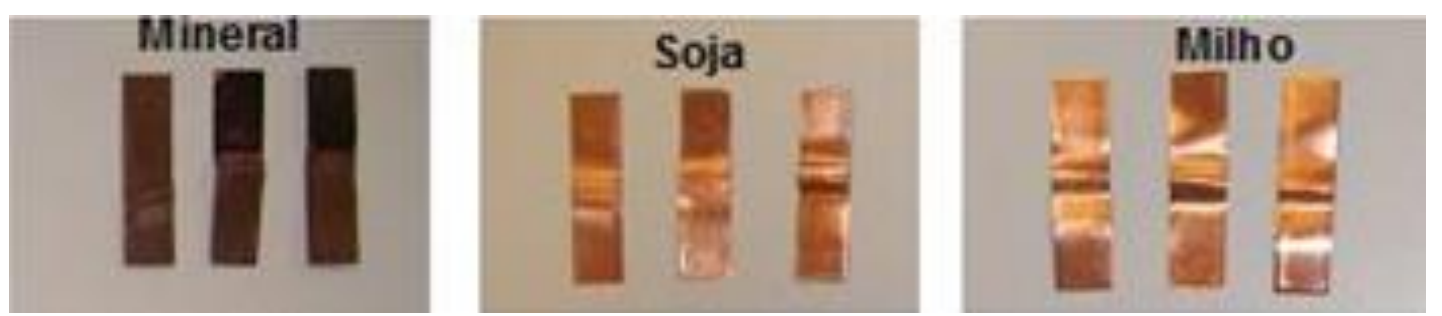

Figura 4. Lâminas de cobre obtidas no ensaio de corrosão.

As lâminas mergulhadas no óleo mineral apresentaram uma coloração que varia de grafite ao negro fosco, sendo classificada como corrosão 4b. Já s lâminas que ficaram mergulhadas nos óleos vegetais apresentaram coloração alaranjado claro, próxima à da fita recém-polida, sendo classificadas como levemente manchada (1a). Assim pode-se dizer que os óleos vegetais não apresentam comportamento corrosivo considerável.

O índice de iodo está relacionado com a quantidade de insaturações presentes, assim um maior índice favorece a ocorrência de processos oxidativos. O óleo mineral apresentou menor quantidade de insaturações, o que era esperado, uma vez que ele é constituído, em sua maioria, de compostos parafínicos. O óleo de soja apresentou maior índice de iodo. Segundo Melo [9], valores elevados para o índice de iodo apesar de torna-o susceptível à degradação térmica e oxidativa, favorece a sua fluidez.

Os valores dos índices de refrações estão de acordo com a literatura, não sendo observadas diferenças significativas nos dados obtidos dos óleos.

Em relação ao ponto de fulgor, os óleos vegetais apresentaram valores muito superiores ao encontrado para o óleo mineral. A margem de segurança aumenta com o aumento do ponto de fulgor do fluido de corte, um indicativo que mostra que a aplicação dos óleos vegetais se torna muito positiva em processos de retificação.

A viscosidade dos fluidos de corte é outro fator importante, uma vez que este parâmetro interfere no desempenho do rebolo durante o processo de retificação. Os óleos vegetais apresentaram resultados correspondentes aos valores médios da literatura. Em relação a densidade os óleos vegetais também apresentaram os

\footnotetext{
* Contribuição técnica ao 69ำ Congresso Anual da ABM - Internacional e ao 14ํㅡㄹ ENEMET - Encontro Nacional de Estudantes de Engenharia Metalúrgica, de Materiais e de Minas,21 a 25 de julho de 2014, São Paulo, SP, Brasil.
} 


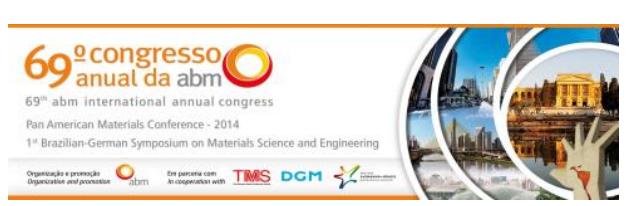

valores médios da literatura. Dentre os óleos vegetais, o óleo de soja foi o que apresentou menores valores para viscosidade e densidade, o que é considerado um resultado favorável para evitar o empastamento do rebolo durante o processo.

A Tabela 3 apresenta as notas atribuídas aos parâmetros avaliativos do desempenho dos fluidos utilizados no processo de retificação.

Tabela 3. Valores atribuídos às análises dos fluidos durante a retificação.

\begin{tabular}{c|c|c|c|c|c|c}
\hline Fluido & Temperatura & Fumaça & Espuma & Odor & Rugosidade & Total \\
\hline Soja & 4 & 4 & 5 & 5 & 5 & 23 \\
\hline Milho & 4 & 4 & 5 & 5 & 3 & 21 \\
\hline Mineral & 4 & 2 & 5 & 1 & 5 & 17 \\
\hline
\end{tabular}

Ao analisar os totais obtidos pela soma das notas atribuídas a cada parâmetro, observa-se que os óleos vegetais apresentaram melhor desempenho que o óleo mineral. Com relação à temperatura, os valores obtidos foram próximos para todos os fluidos testados. Na usinagem com óleo mineral, foi observada a formação de fumaça e odor, o que é ruim, uma vez que causa incômodo ao operador. A ausência de formação de espuma pelos fluidos é positiva, pois a espuma pode dificultar a visibilidade do operador e prejudicar a refrigeração pela formação de bolhas de ar.

Em relação à rugosidade, o óleo mineral e óleo de soja foram os que apresentaram menor rugosidade. Este resultado indica que a substituição do óleo mineral por esta formulação não prejudica o acabamento da peça. Já a substituição pelo óleo de milho apresentou rugosidade superior.

\section{CONCLUSÃO}

A análise dos óleos de soja e milho no processo de retificação apresentaram em geral resultados melhores do que o óleo mineral.

Os valores de rugosidade para o óleo de soja foram próximos ao do óleo mineral, indicando possibilidade de uso deste sem perda na qualidade do acabamento superficial da peça.

O menor índice de acidez dos óleos vegetais acarretou melhores resultados (menor corrosividade) no ensaio de corrosão à lâmina de cobre, sendo este um ponto muito importante para a conservação do equipamento e da peça a ser produzida.

Foi possível observar também que o óleo mineral apresenta baixo ponto de fulgor e viscosidade, quando comparado aos óleos vegetais de uma maneira geral. $\mathrm{O}$ alto ponto de fulgor dos óleos vegetais é um fator interessante ao processo, já que aumenta a margem de segurança do processo de retificação.

Dentre os dois óleos vegetais, o óleo de soja apresentou menores valores para viscosidade e densidade, o que é considerado um resultado favorável para evitar o empastamento do rebolo durante o processo. Porém, ainda assim, o óleo mineral apresenta resultados melhores que os óleos vegetais.

A análise do índice de iodo indica uma maior quantidade de insaturações nos óleos vegetais, o que proporciona uma maior biodegradabilidade aos mesmos, tornandoos ambientalmente amigáveis.

\section{Agradecimentos}

Ao CNPq pela bolsa de Produtividade em Pesquisa, ao CEFET/MG e ao Departamento de Engenharia de Materiais - DEMAT.

\footnotetext{
* Contribuição técnica ao $69^{\circ}$ Congresso Anual da ABM - Internacional e ao 14ํㅡㄹ ENEMET - Encontro Nacional de Estudantes de Engenharia Metalúrgica, de Materiais e de Minas,21 a 25 de julho de 2014, São Paulo, SP, Brasil.
} 


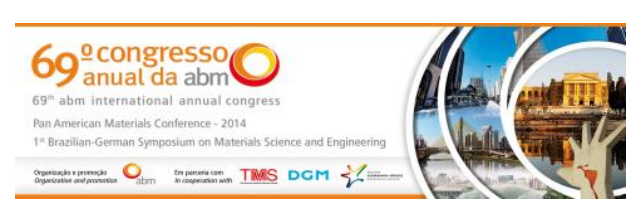

\section{REFERÊNCIAS}

1 Avila RF, Abrão AM. The effect of cutting fluids on the machining of AISI 4340 steel. Journal of Materials Processing Technology. 2001;119:21-26.

2 Maia DAS, Guimarães AP, Araújo RS, Cavalcante Jr. CL, Sant'Ana HB. Fluidos de corte: novas formulações e recuperabilidade. In: Anais do 4ํ PDPETRO; 2007; Campinas, Brasil. Campinas: PDPETRO; 2007. p.1-10.

3 Catai RE. Otimização das condições de refrigeração/lubrificação no processo de retificação cilíndrica de mergulho [tese de doutorado]. Guaratinguetá: Faculdade de Engenharia, Universidade Estadual Paulista; 2004.

4 Alves SM, Oliveira JFG. Vegetable based cutting fluid: an environmental alternative to grinding process. In: Anais da 15 $5^{\underline{a}}$ Conferência Internacional Cirp Em Engenharia Ciclo de Vida; 2008; Sydney, Austrália. Sydney: CIRP; 2008. p.664-668.

5 Runge PRF, Duarte GN. Lubrificantes nas indústrias - produção, manutenção e controle. Cotia: Triboconcept Edições Técnicas; 1990.

6 Zanferrari RB. Opções para trabalhar material endurecido com rebolos galvânicos de CBN. Revista Máquinas e Metais. 2005;472:154-171.

7 Gonçalves JFS. Proposição de método de desenvolvimento de fluido de corte de base vegetal [tese de doutorado]. São José dos Campos: Instituto Tecnológico de Aeronáutica; 2013. p.1-223

8 Fox NJ, Stachowiak GW. Vegetable oil based lubricants- a review of oxidation. Journal Tribol. Int. 2007;40:1035-46.

9 Melo MAMF. Avaliação das propriedades de óleos vegetais visando a produção de biodiesel [dissertação de mestrado]. João Pessoa: Departamento de química, Universidade Federal da Paraíba; 2010.

\footnotetext{
* Contribuição técnica ao 69ำ Congresso Anual da ABM - Internacional e ao 14ํㅡㄹ ENEMET - Encontro Nacional de Estudantes de Engenharia Metalúrgica, de Materiais e de Minas,21 a 25 de julho de 2014, São Paulo, SP, Brasil.
} 